\title{
CENTRAL INTERTWINING NUMBERS FOR REPRESENTATIONS OF FINITE GROUPS
}

BY

\author{
JOHN A. ERNEST( $\left.{ }^{1}\right)$
}

1. Introduction. Let $x \rightarrow L_{x}$ and $x \rightarrow M_{x}$ be two representations of a group $G$. The intertwining vector space of $L$ and $M, \Re(L, M)$ is defined as the set of all bounded linear transformations $T$ from the representation space of $L$ to the representation space of $M$ such that $T L_{x}=M_{x} T$ for all $x$ in $G$. The vector space dimension of $\mathbb{R}(L, M)$ is called the intertwining number of $L$ and $M$ and is denoted $\mathfrak{g}(L, M)$. For finite groups, $\mathfrak{g}(L, L)$ gives the total number of irreducible components appearing in the decomposition of $L$ into irreducible parts, where a component appearing with multiplicity $n$ is counted $n$ times. In particular $L$ is irreducible if and only if $g(L, L)=1$. Intertwining numbers for induced representations are described in [2], and in particular necessary and sufficient conditions are obtained for an irreducible representation to induce an irreducible representation.

In the case $L=M, R(L, L)$ forms a ring and we denote its center by $\mathcal{C} R(L)$. We define the central intertwining number, $\mathfrak{C g}(L)$ of a representation $L$ to be the vector space dimension of $\mathfrak{C} R(L)$. For finite groups $\mathfrak{C} g(L)$ gives the number of distinct irreducible components appearing in the decomposition of $L$ into irreducible parts, where a component appearing with multiplicity $n$ is counted but once. All results on central intertwining numbers should be interpreted from this point of view.

Two representations, $L$ and $M$ are said to be disjoint (denoted $L \delta M$ ) if no subrepresentation of one is equivalent to any subrepresentation of the other. A representation is called a factor representation if it cannot be expressed as the direct sum of two disjoint representations. As is well known and easily proved, a representation $L$ is a factor if and only if $\operatorname{eg}(L)=1$. For finite dimensional representations, a factor is simply some integral multiple of an irreducible representation. For the representation theory of separable locally compact groups, factor representations form a natural "building block." Heuristically, central intertwining numbers give the same type of information with respect to factor representations, as do intertwining numbers with respect to irreducible representations. In this paper we describe the situation only for finite groups, where the phenomenon is purely algebraic and free of measure theoretic difficulties. It is hoped that this will then serve as a useful prolegomena to the more general investigation.

Presented to the Society, January 29, 1960; received by the editors September 30, 1960.

(1) The author is a National Science Foundation fellow. This paper is a revised version of a portion of the author's dissertation, University of Illinois, February, 1960. 
Now that the discussion of motivation is concluded, we restrict ourselves entirely to considerations of finite groups and of finite dimensional representations over an algebraically closed field $\mathcal{F}$ of characteristic zero or of prime characteristic which does not divide the order of the group being represented. If we shift our point of view slightly by considering factor representations as the fundamental objects, we find the classical decomposition theory takes the following form. Let $L$ be a representation of the group $G$ such that $\mathfrak{C g}(L)=n$. Then there exist $n$ projections $E_{1}, E_{2}, \cdots, E_{n}$ in $\mathcal{C} R(L)$ such that $E_{i} E_{j}=E_{j} E_{i}=\delta_{i j} E_{j}, \sum_{i=1}^{n} E_{i}=I$, and the set $\left\{E_{j}\right\}$ forms a vector space basis for $\mathcal{C} R(L)$. Further $L=\sum_{i=1}^{n} L^{(i)}$ where $L^{(i)}$ is the restriction of $L$ to the range of $E_{i}$. Each $L^{(i)}$ is a factor representation and $L^{(i)}$ and $L^{(j)}$ are disjoint if $i \neq j$. Further this decomposition is unique up to equivalence and up to the order in which the summands occur. From this we see that $\mathfrak{C} g(L)$ is equal to the number of disjoint factor representations which are subrepresentations of $L$ and that $\mathfrak{C g}$ is additive over disjoint representations.

In section 2 we characterize $\mathcal{C} R(L)$ as the vector space generated by a specific set of elements. Thus the determination of $\mathfrak{C g}(L)$ is reduced to determining the maximal number of these generating elements which are linearly independent. This approach is used in section 3 to determine the central intertwining number of an induced representation. In particular we obtain a necessary and sufficient condition for a factor representation to induce a factor representation.

2. Characterization of $\mathbb{e} R(L)$. If $H$ is a subgroup of the group $G, L$ is a representation of $H$, and $r \in G$, then $L^{r}$, the conjugate representation of $L$ with respect to $r$, is defined as the representation of $r^{-1} H r$ given by $L_{x}^{r}=L_{r x r^{-1}}$ for each $x \in r^{-1} H r$. If $L$ is a representation of a group $G$ and $C$ is a class (of conjugates) of $G$, then we define the corresponding $L$-conjugate sum to be $S=\sum_{x \in C} L_{x}$.

Theorem 1. Let $L$ be a representation of the group $G$ and let $C_{1}, \cdots, C_{m}$ denote the distinct classes (of conjugates) of $G$. Let $S_{1}, \cdots, S_{m}$ denote the corresponding L-conjugate sums. Then the set $\left\{S_{1}, \cdots, S_{m}\right\}$ generates $\mathcal{C} R(L)$ as a vector space.

Proof. The reader may verify that $T \in \mathcal{C} R(L)$ if and only if $T$ is of the form $\sum_{i=1}^{n} \alpha_{i} L_{x_{i}}$ for some $\alpha_{i} \in \mathcal{F}$, some $x_{i} \in G, i=1, \cdots, n$, and has the property that

$$
\sum_{i=1}^{n} \alpha_{i} L_{x_{i}}=\sum_{i=1}^{n} \alpha_{i} L_{x_{i}}^{y}
$$

for all $y$ in $G$. Here $L^{y}$ denotes the conjugate representation of $L$, with respect to $y$, defined by $L_{x}^{y}=L_{y x y^{-1}}$ for each $x \in G$. Thus each $L$-conjugate sum $S_{i}$ is contained in $\mathcal{C} R(L)$. Suppose $T=\sum_{i=1}^{n} \alpha_{i} L_{x_{i}}$ is an arbitrary element of 
$\mathcal{C} R(L)$, where $\alpha_{i} \in F$ and $x_{i} \in G$. Then by the previous remark, for each $y \in G, T=\sum_{i=1}^{n} \alpha_{i} L_{x_{i}}^{y}$. Let $g$ denote the order of $G$. Then

$$
T=(1 / g) \sum_{y \in G} \sum_{i=1}^{n} \alpha_{i} L_{x_{i}}^{y}=\sum_{i=1}^{n}\left(\alpha_{i} / g\right) \sum_{y \in G} L_{x_{i}}^{y} .
$$

Let $C_{i}$ denote the class to which $x_{i}$ belongs. (This will, in general, require a reindexing of the classes. Some classes may not appear in the new enumeration, while others may appear more than once.) The reader may verify that the number $\beta_{i}$, of elements in the set $\left\{y: y \in G\right.$ and $\left.y x_{i} y^{-1}=z\right\}$, where $z \in C_{i}$, does not depend on the choice of the element $z \in C_{i}$. Then $g=\beta_{i} \gamma_{i}$ where $\gamma_{i}$ denotes the number of elements in $C_{i}, 1 \leqq i \leqq n$. Hence we may write

Thus

$$
\sum_{y \in G} L_{x_{i}}^{y}=\beta_{i} \sum_{x \in C_{i}} L_{x}=\left(g / \gamma_{i}\right) S_{i}
$$

$$
T=\sum_{i=1}^{n}\left(\alpha_{i} / g\right) \sum_{y \in G} L_{x_{i}}^{y}=\sum_{i=1}^{n}\left(\alpha_{i} / \gamma_{i}\right) S_{i} .
$$

The theorem just proved implies the well-known result that $\operatorname{eg}(L)$ is always less than or equal to the number of classes of $G$. In particular if $L$ is the regular representation of $G$ it is immediate that the $L$-conjugate sums are linearly independent, which gives the well-known fact that the central intertwining number of the regular representation is equal to the number of classes of $G$.

3. Induced representations. We first state the usual definition of induced representation, with a modification of the usual notation.

Case 1 . Let $H$ be a subgroup of $G$ and $M$ a representation of $G$. Then we define $M \mid H$ (read: the representation which $M$ induces in the subgroup $H$ ) to be the restriction of $M$ to $H$, i.e., $(M \mid H)_{x}=M_{x}$ for each $x \in H$.

Case 2. Let $H$ be a subgroup of $G$ and $L$ a representation of $H$. We define $G \mid L$ (read: the representation of $G$ induced by $L$ ) as follows. Let $\mathfrak{H}(G \mid L)$ denote the set of all functions $f$ on $G$, taking values in the representation space $\mathfrak{H C}(L)$ of $L$ and which satisfy $f(x r)=L_{x} f(r)$, for $x \in H$ and $r \in G$. $\mathfrak{H C}(G \mid L)$ is given a vector space structure in the usual way and we define, for each $x \in G$, $\left[(G \mid L)_{x} f\right](y)=f(y x)$ for each $f \in \mathcal{H}(G \mid L)$ and $y \in G$.

The notation introduced is designed to mirror the "reciprocity" between these two types of inducing.

In the general case the problem of determining the exact decomposition of an induced representation into irreducible parts is a difficult and unsolved problem. The work of Professor Mackey in [2] gives information as to the total number of irreducible parts which will appear in such a decomposition. The following considerations are designed to give the additional information as to the maximal number of distinct irreducible representations which appear. 
We first consider the case of a representation induced in a subgroup (cf. [1]). Let $L$ be a representation of a finite group $G$ and let $H$ be a subgroup of $G$. To determine $\operatorname{eg}(L \mid H)$, one considers the classes $C_{1}, \cdots, C_{m}$ of $H$ and forms the corresponding $(L \mid H)$-conjugate sums $S_{i}=\sum_{x \in C_{i}} L_{x}$. The problem is thus reduced to determining the maximal number of these $S_{i}$ which are linearly independent. As an illustration of this procedure we prove the following theorem.

THEOREM 2. Let $L$ be a factor (or irreducible) representation of a group $G$. Let $H$ be a subgroup of $G$. Let $\alpha$ denote the number of classes of $H$ and $\beta$ the number of classes of $G$ which are completely contained in $H$. Then the number of distinct irreducible components appearing in the decomposition of $L \mid H$ is less than or equal to $\alpha-\beta+1$.

Proof. Let $C_{1}, \cdots, C_{\alpha}$ denote the classes of $H$ and $D_{1}, \cdots, D_{\beta}$ the classes of $G$ completely contained in $H$. Choose $D_{\beta}=C_{\alpha}=e$, where $e$ is the identity element of $G$. Since $L$ is a factor representation we have that $\sum_{x \in D_{i}} L_{x}$ $=k_{i} I$, for some $k_{i} \in \mathcal{F}, i=1, \cdots, \beta$, where $I$ is the identity operator. Each $D_{i}$ is the union of some subcollection $\left\{C_{i j}: j=1, \cdots, m_{i}\right\}$ of the classes of $H$. Then

$$
\sum_{x \in D_{i}} L_{x}=\sum_{j=1}^{m_{i}} \sum_{x \in C_{i j}} L_{x}=k_{i} I=k_{i} \sum_{x \in C_{\alpha}} L_{x}
$$

which gives a dependency relation among the $(L \mid H)$-conjugate sums corresponding to the $C_{i j}$. Thus one of these $(L \mid H)$-conjugate sums can be eliminated from the generating set, for each $i=1, \cdots, \beta-1$. We are thus left with a generating set of $\alpha-(\beta-1)$ elements. Hence $\operatorname{eg}(L \mid H) \leqq \alpha-\beta+1$.

Additional information about $\operatorname{eg}(L \mid H)$ may be obtained from the classical theory. Again let $L$ be an irreducible representation of a finite group $G$ and let $H$ be a subgroup of $G$. Then $\operatorname{dim}(L \mid H)=\operatorname{dim}(L)$. Suppose $M$ is an irreducible component of $L \mid H$. By the Frobenius reciprocity theorem, $G \mid M$ must contain $L$ and thus $\operatorname{dim}(G \mid M)=[G: H] \operatorname{dim}(M) \geqq \operatorname{dim}(L)=\operatorname{dim}(L \mid H)$. Thus $L \mid H$ can have at most $[G: H]$ irreducible components. Hence $\operatorname{Cg}(L \mid H)$ $\leqq g(L|H, L| H) \leqq[G: H]$. From this we conclude that if $L$ is any representation of $G$, then $\operatorname{eg}(L \mid H) \leqq[G: H] \operatorname{eg}(L)$. In particular if we take $L$ to be the regular representation of $G$, we obtain the purely group theoretical fact that the number of classes of $H$ is less than or equal to the number of classes of $G$ times $[G: H]$.

We next turn our attention to Case 2, representations induced in a supergroup.

THEOREM 3. Let $H$ be a subgroup of $G$ and let $L$ be a representation of $H$. Let $D_{1}, \cdots, D_{n}$ denote the classes of $G$, and let $S_{1}, \cdots, S_{n}$ denote the corresponding $(G \mid L)$-conjugate sums. Then $T=\sum_{i=1}^{n} \alpha_{i} S_{i}=0$ for some $\alpha_{i} \in \mathcal{F}$ if and only if, for each $r$ in $G$, 


$$
\sum_{i=1}^{n} \alpha_{i} \sum_{x \in r D_{i} \cap H} L_{x}=0
$$

(Summations over empty sets are defined to be equal to 0.)

Proof. For each $t$ in $G$ and $f$ in $\operatorname{HC}(G \mid L)$ we have

$$
\lfloor T f](t)=\left[\sum_{i=1}^{n} \alpha_{i} \sum_{s \in D_{i}}(G \mid L)_{s} f\right](t)=\sum_{i=1}^{n} \alpha_{i} \sum_{s \in t D_{i}} f(s) .
$$

Let $\sigma_{1}, \cdots, \sigma_{k}$ denote representatives of the right $H$-cosets of $G$, where $k=[G: H]$ and let $v_{1}, \cdots, v_{m}$ be a basis for $\mathcal{H C}(L)$. We now define a basis $\left\{f_{a b}\right\}, a=1, \cdots, k ; b=1, \cdots, m$, for $\mathfrak{H C}(G \mid L)$ as follows. Let $f_{a b}\left(\sigma_{a}\right)=v_{b}$, for $a=1, \cdots, k ; b=1, \cdots, m, f_{a b}\left(\sigma_{i}\right)=0$ if $a \neq i, 1 \leqq a, i \leqq k$, and finally $f_{a b}\left(x \sigma_{i}\right)$ $=L_{x} f_{a b}\left(\sigma_{i}\right)$, for each $x$ in $H, 1 \leqq a, i \leqq k, 1 \leqq b \leqq m$. Then $T=0$ if and only if, for each $t$ in $G$

$$
\sum_{i=1}^{n} \alpha_{i} \sum_{s \in t D_{i}} f_{a b}(s)=0
$$

for $a=1, \cdots, k ; b=1, \cdots, m$. The expression on the left is equal to

$$
\begin{aligned}
\sum_{i=1}^{n} \alpha_{i} \sum_{a \in t D_{i} \cap \cap_{H a}} f_{a b}(s) & =\sum_{i=1}^{n} \alpha_{i} \sum_{x \in t \sigma_{a}^{-1} D_{i} \cap H} L_{x} f_{a b}\left(\sigma_{a}\right) \\
& =\left[\sum_{i=1}^{n} \alpha_{i} \sum_{x \in t \sigma_{a}^{-1} \cdot D_{i} \cap \cap_{H}} L_{z}\right] v_{b}
\end{aligned}
$$

Thus $T=0$ if and only if, for each $r \in G$,

$$
\sum_{i=1}^{n} \alpha_{i} \sum_{x \in r D_{i} \cap_{H}} L_{x}=0
$$

Corollary. Let $H, G, L, D_{i}, S_{i}$ and $T$ be as in the statement of Theorem 3. Let $[G: H]=k$ and let $\sigma_{1}, \cdots, \sigma_{k}$ denote representatives of the right $H$-cosets of $G$, (or of the left $H$-cosets of $G$ ). Then $T=0$ if and only if, for $j=1, \cdots, k$,

$$
\sum_{i=1}^{n} \alpha_{i} \sum_{x \in \sigma_{j} D_{i} \cap_{H}} L_{x}=0
$$

Proof. Left to the reader.

Theorem 3, with its corollary, gives a straightforward method of computing $\operatorname{eg}(G \mid L)$ when the classes of $G$ are known. This result, together with Theorem 1, reduces the problem of computing $\operatorname{eg}(G \mid L)$ to the problem of determining whether or not a certain system of homogeneous linear equations has a nontrivial solution. 
While the basic phenomena are described in Theorem 3 and its corollary, the result may be stated in various ways. We use the notation introduced in the statement of the corollary to Theorem 3. Let

$$
R_{i j}=\sum_{x \in \sigma_{j} D_{i} \cap_{H}} L_{x}=\sum_{x \in D_{i} \sigma_{j} \cap_{H}} L_{x}
$$

$i=1, \cdots, n ; j=1, \cdots, k$. Then $\left\{\left(R_{i 1}, R_{i 2}, \cdots, R_{i k}\right): i=1, \cdots, n\right\}$ is a set of $k q^{2}$-dimensional vectors over $\mathcal{F}$, where $q=\operatorname{dim}(L)$. Then $\operatorname{eg}(G \mid L)$ is equal to the maximal number of these vectors which are linearly independent over $\mathcal{F}$. This result takes a still simpler form if $L$ is linear, i.e., one-dimensional. Then $\left(R_{i j}\right), 1 \leqq i \leqq n, 1 \leqq j \leqq k$, is an $n \times k$ matrix over $\mathcal{F}$ and $\operatorname{eg}(G \mid L)$ is the rank of this matrix.

The following theorem will illustrate how the criterion of Theorem 3 may be used in the calculation of central intertwining numbers.

Theorem 4. Suppose that $H$ is a normal subgroup of $G, k=[G: H]$ and that $\left\{H \sigma_{i}\right\}, i=1, \cdots, k$, are the $H$-cosets of $G$. Further suppose that, for each $i=1, \cdots, k$, the inner automorphism of $H$ determined by $\sigma_{i}$ leaves the classes of $H$ fixed. If $\sigma_{i}$ is contained in the center of $G$, for $i=1, \cdots, n,(n \leqq k)$, then $\mathfrak{e g}(G \mid L) \geqq n \mathfrak{e g}(L)$.

Proof. Let $\mathrm{eg}(L)=c$. Index the classes $\left\{C_{1}, \ldots, C_{m}\right\}$ of $H$ in such a way that the corresponding $L$-conjugate sums $T_{i}=\sum_{x \in C_{i}} L_{x}, i=1, \cdots, c$, are linearly independent over $\mathcal{F}$. Let $S_{i j}=\sum_{s \in C_{i} \sigma_{j}}(G \mid L)_{s}$. We leave it to the reader to verify that under the hypothesis of the theorem, the $C_{i} \sigma_{j}$ are classes of $G$, for $1 \leqq i \leqq c, 1 \leqq j \leqq n$, and thus that $S_{i j} \in \mathcal{C} R(G \mid L)$ for $1 \leqq i \leqq c, 1 \leqq j \leqq n$. We next show that the $S_{i j}$, for $1 \leqq i \leqq c, 1 \leqq j \leqq n$, are linearly independent over F. Suppose $\sum_{i=1}^{c} \sum_{j=1}^{n} \alpha_{i j} S_{i j}=0$, where $\alpha_{i j} \in \mathcal{F}$. By Theorem 3 this implies that, for each $r \in G$,

$$
\sum_{i=1}^{c} \sum_{j=1}^{n} \alpha_{i j} \sum_{n \in C_{i} \sigma_{j} \cap_{H}} L_{x}=0 .
$$

Taking $r=\sigma_{j}^{-1}$ this becomes

$$
\sum_{i=1}^{c} \alpha_{i j} \sum_{x \in C_{i}} L_{x}=\sum_{i=1}^{c} \alpha_{i j} T_{i}=0
$$

for $j=1, \cdots, n$. By the linear independence of the $T_{i}$, we conclude that $\alpha_{i j}=0,1 \leqq i \leqq c, 1 \leqq j \leqq n$. Hence $\mathcal{e g}(G \mid L) \geqq n c$.

We next concentrate on the situation where $H$ is a normal subgroup of $G$. Two representations $L$ and $M$ of $H$ are said to be conjugately disjoint relative to $G$, denoted $L \bar{\delta} M$, if every conjugate of $L$, relative to $G$, is disjoint from every conjugate of $M$, relative to $G$. $L$ and $M$ are said to be in the same orbit of $H$, relative to $G$, if $L$ is equivalent to a conjugate of $M$, relative to $G$. Now suppose $L$ is a representation of $H$ which has the form $L=a_{1} L_{1}+\cdots+a_{n} L_{n}$, 
where the $a_{i}$ are positive integers and the $L_{i}$ are mutually disjoint irreducible representations of $H$. Group the set $\left\{L_{1}, \cdots, L_{n}\right\}$ into orbits, relative to $G$. We define the conjugacy number of $L$, denoted $\operatorname{er}(L)$, to be the number of these orbits.

Clearly $\mathfrak{C} g(L) \geqq \mathfrak{C} \mathfrak{N}(L)$. The corollary to Theorem 10 of [4] states that if $H$ is a normal subgroup of $G$ and if one groups the set of all irreducible representations of $H$ into orbits, relative to $G$, then the number of such orbits is equal to the number of classes of $G$ which are contained in $H$. (Also see [5].) Thus for any representation $L$ of $H, \mathfrak{e} \Re(L)$ is less than or equal to the number of classes of $G$ which are contained in $H$.

TheOREM 5. Suppose that $H$ is a normal subgroup of $G$ and that $L$ is a representation of $H$. Let $L=M_{1}+\cdots+M_{n}$ where $n=\operatorname{eg}(L)$ and each $M_{i}$ is a linear combination, with positive integral coefficients, of irreducible representations of $H$, all belonging to the same orbit $C_{i}$. Then $\operatorname{eg}(G \mid L)=\sum_{i=1}^{n} \operatorname{eg}\left(G \mid M_{i}\right)$ and $\operatorname{eg}\left(G \mid M_{i}\right)=\operatorname{eg}\left(G \mid L_{i}\right)$ where $L_{i}$ is any element of $C_{i}$.

Proof. Since $M_{i} \bar{\delta} M_{j}$ if $i \neq j$, we have, by Corollary 7, page 129 of [3], that $\left(G \mid M_{i}\right) \diamond\left(G \mid M_{j}\right)$ if $i \neq j$. Hence, since $\mathrm{eg}$ is additive over disjoint representations, we have $\operatorname{eg}(G \mid L)=\operatorname{eg}\left(G\left|M_{1}+\cdots+G\right| M_{n}\right)=\operatorname{eg}\left(G \mid M_{1}\right)+\cdots$ $+\operatorname{eg}\left(G \mid M_{n}\right)$. Now suppose that $M_{i}=a_{1} L_{i 1}+\cdots+a_{m} L_{i m}$ where the $a_{j}, j=1, \cdots, m$, are positive integers and the $L_{i j}, j=1, \cdots, m$, are irreducible representations, all in the same orbit $C_{i}$, relative to $G$. Then $\operatorname{eg}\left(G \mid M_{i}\right)=\mathfrak{e g}\left(G\left|L_{i 1}+\cdots+G\right| L_{i m}\right)$. However, as the reader may easily verify, conjugate representations induce equivalent representations and thus $G\left|L_{i j} \simeq G\right| L_{i}$ for $j=1, \cdots, m$, where $L_{i}$ is any element of $C_{i}$. Hence $\operatorname{eg}\left(G \mid M_{i}\right)$ $=\mathfrak{e g}\left(m\left(G \mid L_{i}\right)\right)=\mathfrak{C g}\left(G \mid L_{i}\right)$.

Corollary. Let $H$ be a normal subgroup of $G$ and let $C_{1}, \cdots, C_{n}$ denote the orbits of irreducible representations of $H$, relative to $G$. If $L$ is a representation of $H$ such that $\operatorname{ex}(L)=n$, then $\operatorname{eg}(G \mid L)$ is equal to the number of classes of $G$.

Proof. Let $M$ denote the regular representation of $H$. Then $G \mid M$ is the regular representation of $G$ and it suffices to prove that $\operatorname{eg}(G \mid L)=\operatorname{Cg}(G \mid M)$. However, we have $L=\sum_{i=1}^{n} L_{i}$ and $M=\sum_{i=1}^{n} M_{i}$ where both $L_{i}$ and $M_{i}$ are linear combinations of elements of $C_{i}$, with positive integral coefficients, for $i=1, \cdots, n$. The stated result now follows from the above theorem.

We next obtain an upper bound on $\operatorname{eg}(G \mid L)$, analogous to that obtained for representations induced in a subgroup.

THEOREM 6. Let $H$ be a subgroup of $G$ and $L$ a representation of $H$. Then $\operatorname{eg}(G \mid L) \leqq[G: H] \operatorname{eg}(L)$. Further if $H$ is a normal subgroup, then $\operatorname{eg}(G \mid L)$ $\leqq[G: H] \operatorname{er}(L)$.

Proof. First take $L$ to be irreducible. Suppose that $M$ is an irreducible component of $G \mid L$. Then, by the Frobenius reciprocity theorem, $L$ appears 
as a component of $M \mid H$. Hence $\operatorname{dim}(M)=\operatorname{dim}(M \mid H) \geqq \operatorname{dim}(L) . \operatorname{But} \operatorname{dim}(G \mid L)$ $=[G: H] \operatorname{dim}(L)$. Thus $(G \mid L)$ has at most $[G: H]$ irreducible components. Hence $\operatorname{eg}(G \mid L) \leqq[G: H]$, which easily generalizes to the statement of the theorem when $L$ is not irreducible.

Now suppose $H$ is a normal subgroup of $G$ and $L$ is a representation of $H$. We may write $L=M_{1}+\cdots+M_{n}$ where $n=\mathfrak{e} \mathfrak{N}(L)$ and each $M_{i}$ is a linear combination, with positive integral coefficients, of irreducible representations, all of which are in the same orbit of $H$ relative to $G$. Then by Theorem 5 we have that $\operatorname{eg}(G \mid L)=\operatorname{eg}\left(G \mid M_{1}\right)+\cdots+\mathfrak{C g}\left(G \mid M_{n}\right)$ and $\operatorname{eg}\left(G \mid M_{i}\right)$ $=\operatorname{eg}\left(G \mid L_{i}\right)$, where $L_{i}$ is any irreducible representation appearing in the decomposition of $M_{i}, i=1, \cdots, n$. Hence, by the first part of the theorem we have, $\operatorname{eg}(G \mid L)=\operatorname{eg}\left(G \mid L_{1}\right)+\cdots+\operatorname{eg}\left(G \mid L_{n}\right) \leqq n[G: H]=[G: H] \mathfrak{e r}(L)$.

CoROllary 1. If $H$ is a subgroup of $G$, then the number of classes of $G$ is less than or equal to $[G: H]$ times the number of classes of $H$.

Corollary 2. Suppose that $H$ is a normal subgroup of $G$ and that every $H$ coset of $G$ has a nonempty intersection with the center of $G$. If $L$ is a representation of $H$, then $\operatorname{eg}(G \mid L)=[G: H] \operatorname{eg}(L)$.

Proof. By Theorem 4 we have $\operatorname{eg}(G \mid L) \geqq[G: H] \mathfrak{e g}(L)$.

CoRollary $3\left(^{2}\right)$. Suppose that $H$ is a normal subgroup of $G$ and that every $H$-coset of $G$ has a nonempty intersection with the center of $G$. If $L$ is an irreducible representation of $H$, then $G \mid L=L_{1}+\cdots+L_{k}$, where the $L_{i}$ are mutually disjoint irreducible representations such that $L_{i} \mid H=L$ for $i=1, \cdots, k$, where $k=[G: H]$.

Proof. By Corollary 2 we have that $\operatorname{eg}(G \mid L)=[G: H]$ and thus $G \mid L$ $=a_{1} L_{1}+\cdots+a_{k} L_{k}$ where the $a_{i}$ are positive integers and the $L_{i}$ are mutually disjoint irreducible representations. By the Frobenius reciprocity theorem, $L_{i} \mid H$ must contain $L$ and thus $\operatorname{dim}\left(L_{i}\right) \geqq \operatorname{dim}(L)$. Further we have $\operatorname{dim}(G \mid L)=[G: H] \operatorname{dim}(L)=k \operatorname{dim}(L)$. Hence $a_{1}=\cdots=a_{k}=1$ and $\operatorname{dim}\left(L_{i}\right)$ $=\operatorname{dim}(L)$. Since $L_{i} \mid H$ must contain $L$, we have $L_{i} \mid H=L$.

COROLlaRY 4. Let $H$ be a normal subgroup of $G$. Then the number of classes of $G$ is less than or equal to $[G: H]$ times the number of classes of $G$ which are contained in $H$.

Proof. As we remarked earlier, the corollary to Theorem 10 of [4] implies that, for any representation $L$ of $H, \mathfrak{e} \mathfrak{N}(L)$ is less than or equal to the number of classes of $G$ which are contained in $H$. (See also [5].) But if $L$ is the regular representation of $H$, then $G \mid L$ is the regular representation of $G$ and thus the number of classes of $G$ is equal to $\operatorname{eg}(G \mid L)$, which is less than or equal to $[G: H] \mathfrak{e} \Re(L)$.

(2) This corollary was suggested to the author by Professor M. Suzuki. 
THEOREM 7. Let $H$ be a subgroup of $G$ and let $L$ be a factor representation of $H$. Then $G \mid L$ is a factor representation if and only if, for each class $D$ of $G$,

$$
\sum_{x \in r D \cap_{H}} L_{x}=0
$$

for all $r \in G$ such that $r \notin H$.

Proof. By Theorem $1, G \mid L$ is a factor representation if and only if, for each class $D$ of $G$, there exists an $\alpha \in \mathcal{F}$, such that $S+\alpha I=0$, where $S$ is the $(G \mid L)$-conjugate sum corresponding to $D$, and $I=(G \mid L)_{e}$ is the identity operator on $\operatorname{HC}(G \mid L)$. By Theorem 3 there exists such an $\alpha$ if and only if

$$
\sum_{x \in r D \cap_{H}} L_{x}+\alpha \sum_{x \in r\{e\} \cap_{H}} L_{x}=0
$$

for each $r \in G$. If $r \notin H$, then $r\{e\} \cap H=\{r\} \cap H$ is empty. Thus there exists an $\alpha \in \mathcal{F}$ such that $S+\alpha I=0$ if and only if

$$
\sum_{x \in r D \cap_{H}} L_{x}=0
$$

for all $r$ in $G$ such that $r \notin H$, and an $\alpha$ exists in $\mathcal{F}$ such that

$$
\sum_{x \in y D \cap_{H}} L_{x}+\alpha L_{y}=0
$$

for all $y$ in $H$.

Let $T$ denote the summation of $L_{x}$ over the set $D \cap H$. Then condition (ii) is equivalent to the condition that $T$ be a scalar multiple of $I$. However, since $D \cap H$ is normal in $H$, it is easily verified that $T \in \mathbb{C} R(L)$. The hypothesis that $L$ is a factor representation thus implies that $T$ is a scalar multiple of $I$. Thus there exists an $\alpha \in \mathcal{F}$ such that $S+\alpha I=0$ if and only if condition (i) holds.

CoRollary 1. Let $H$ be a subgroup of $G$ and let $L$ be a factor representation of $H$. Let $e, \sigma_{2}, \cdots, \sigma_{k}$ denote a set of representatives of the right $H$-cosets of $G$ (or of the left $H$-cosets of $G$ ). Then $G \mid L$ is a factor representation if and only if, for each class $D$ of $G$,

$$
\sum_{x \in \sigma_{i} D \cap_{H}} L_{x}=0, \quad \text { for } i=2, \cdots, k
$$

Proof. Left to the reader.

COROLlaRY 2. Let $H$ be a normal subgroup of $G$ and let $L$ be a factor representation of $H$. Then $G \mid L$ is a factor representation if and only if, for each class $D$ of $G$ such that $D \nsubseteq H$, and for each $r$ in $G, \sum_{x \in r D \cap_{H}} L_{x}=0$.

Proof. If $r \in H$, and $D \nsubseteq H$, then $r D \cap H$ is empty. If $D \subset H$ and $r \notin H$, then $r D \cap H$ is empty. 
Corollary 3. Suppose $H$ is a subgroup of $G$ and suppose there exists a factor representation $L$ of $H$ such that $G \mid L$ is a factor representation. Then $H$ contains the center of $G$.

Proof. Suppose that $s$ is contained in the center of $G$ and that $s \notin H$. Then $D=\{s\}$ is a class of $G$, and thus by Theorem 7 ,

$$
0=\sum_{x \in x^{-1}\{s\} \cap_{H}} L_{x}=L_{s}=I,
$$

which is a contradiction. (Compare this result with Corollary 2 to Theorem 5.)

Theorem 7 is the analogue of the Mackey-Shoda theorem (Theorem 6 of [2]) which gives a necessary and sufficient condition for an irreducible representation to induce an irreducible representation. The analogy between these two situations breaks down at one point in that for an induced representation $G \mid L$ to be irreducible, it is necessary that the representation $L$ be irreducible. However it is possible for $G \mid L$ to be a factor representation, without $L$ being a factor representation. It is necessary that $\mathfrak{C} \Re(L)=1$.

The Mackey-Shoda theorem states that if $H$ is a subgroup of $G$ and $L$ is an irreducible representation of $H$, then $G \mid L$ is irreducible if and only if $\left(L \mid r^{-1} H r \cap H\right) \delta\left(L^{r} \mid r^{-1} H r \cap H\right)$ for all $r \in G$ such that $r \notin H$. This condition is clearly a sufficient condition for a factor representation $L$ of $H$ to induce a factor representation $G \mid L$. One sees directly that the Mackey-Shoda condition implies the condition of Theorem 7 by verifying that if $r \in G, r \notin H$, and $D$ is a class of $G$, then

$$
\sum_{x \in r D \cap_{H}} L_{x} \in \mathcal{R}\left(L\left|r^{-1} H r \cap H, L^{r}\right| r^{-1} H r \cap H\right) .
$$

\section{BiBLIOGRAPHY}

1. A. H. Clifford, Representations induced in an invariant subgroup, Ann. of Math. vol. 38 (1937) pp. 533-550.

2. G. W. Mackey, Induced representations of groups, Amer. J. Math. vol. 73 (1951) pp. 576592.

3. - The theory of group representations, University of Chicago mimeographed notes, 1955.

4. T. Nakayama, Some studies on regular representations, induced representations and modular representations, Ann. of Math. vol. 39 (1938) pp. 361-369.

5. T. Osima, Beweis eines Satzes in der Darstellungstheorie, Proc. Japan Acad. vol. 13 (1937) pp. 121-124.

The Institute for Advanced Study, Princeton, New Jersey

UNIVERSITY OF ILLINOIS, URBANA, ILLINOIS 